\title{
Journal statistics, directions for development, and appreciation for reviewers
}

\author{
Hyun Young Koo \\ Professor, College of Nursing · Research Institute of Nursing Science, Daegu Catholic University, Daegu, Korea
}

All manuscripts published in Child Health Nursing Research (CHNR) are indexed and tracked by Scopus, Directory of Open Access Journals (DOAJ), Cumulative Index to Nursing and Allied Health Literature (CINAHL), Google Scholar, Crossref, Korea Citation Index (KCI), KoreaMed, and Korean Medical Citation Index (KoMCI). CHNR was accepted for inclusion in Scopus by the Scopus Content Selection and Advisory Board (CSAB) in February 2018, and it was accepted for inclusion in DOAJ in July 2019. In addition CHNR was re-evaluated by the National Research Foundation of Korea (NRF) in 2020, and has been included in the KCI. All of the manuscripts in CHNR are freely available with open access for everyone to read and download from the CHNR website (http://www.e-chnr.org/) immediately and permanently after publication.

In 2020, a total of 46 articles were published in the four issues of volume 26 of CHNR, of which 29 were written in English and 17 in Korean (Table 1). The average number of authors was 2.8 persons $(\mathrm{SD}=1.5$, range $=1-6)$, and $83 \%$ of the authors were Korean, while 17\% were North American, Asian, or European. By contrast, an analysis of the 146 articles published in CHNR during the 3-year period between 2016 and 2018 revealed that all of the authors were Korean [1]. It is our understanding that the inclusion of CHNR in international databases in turn led to an increase in international submissions. Furthermore, $45.7 \%$ of the research was funded, and approximately $50 \%$ of the funding was provided by national institutions.

\section{Corresponding author Hyun Young Koo \\ https://orcid.org/0000-0001-5848-2143}

College of Nursing, Daegu Catholic University,

33 Duryugongwon-ro, 17-gil, Nam-gu, Daegu 42472, Korea

TEL +82-53-650-4829 FAX +82-53-650-4392

E-MAIL hykoo@cu.ac.kr

Received Jan 19, 2021 Accepted Jan 20, 2021

(a) This is an Open Access article distributed under the terms of the Creative Commons Attribution NonCommercial License (http://creativecommons.org/licenses/by-nc/4.0/) which permits unrestricted noncommercial use, distribution, and reproduction in any medium, provided the original work is properly cited.
Table 1. Bibliometric Analysis of Child Health Nursing Research in $2020(N=46)$

\begin{tabular}{lrl}
\hline Categories & $\mathrm{n}(\%)$ or M \pm SD & Range \\
\hline Number of manuscripts & \\
$26(1)$ & $13(28.2)$ \\
$26(2)$ & $8(17.4)$ \\
$26(3)$ & $8(17.4)$ \\
$26(4)$ & \\
Language of manuscripts & $17(37.0)$ \\
Korean & $29(63.0)$ \\
English & $2.8 \pm 1.5$ \\
Number of authors & \\
Countries of authors* & $44(83.0)$ \\
Korea & $2(3.7)$ \\
United States & $1(1.9)$ \\
Indonesia & $1(1.9)$ \\
Malaysia & $1(1.9)$ \\
Sri Lanka & $1(1.9)$ \\
Nepal & $1(1.9)$ \\
Thailand & $1(1.9)$ \\
Netherlands & $1(1.9)$ \\
Philippines & \\
Research funding & $25(54.3)$ \\
Not supported & $21(45.7)$ \\
Supported & $10(47.6)$ \\
- By a national institution & $8(38.1)$ \\
- By a university & $3(14.3)$ \\
$\quad$ - By a public foundation & \\
\hline
\end{tabular}

*Multiple responses.

The most common study subjects were school-age children (18.3\%), followed by adolescents and parents (16.7\% each) (Table 2). In $65 \%$ of the studies, the subjects were children ranging from newborns to adolescents. Similarly, $60.5 \%$ of the studies published in CHNR in 2014 had children as subjects [2]. Surveys were the most frequently used research method (50.0\%), and survey-based research accounted for a similar proportion of articles published in 2014 (44.7\%) [2] and 20162018 (48.8\%) [1]; as such, over the last 7 years, nearly half of 
Table 2. Research Participants and Research Design of Studies Published in Child Health Nursing Research in 2020 (N=46)

\begin{tabular}{llc}
\hline Variables & Categories & $\mathrm{n}(\%)$ \\
\hline Research & Newborns & $6(10.0)$ \\
participants* & Infants and toddlers & $8(13.3)$ \\
& Preschoolers & $4(6.7)$ \\
& School-age children & $11(18.3)$ \\
& Adolescents & $10(16.7)$ \\
& Young adults & $5(8.3)$ \\
& Parents & $10(16.7)$ \\
Research & Health care providers & $6(10.0)$ \\
methods & Surveys & $23(50.0)$ \\
& Instrument development & $2(4.3)$ \\
& Program development and & $6(13.0)$ \\
& intervention & \\
& Literature review & $6(13.0)$ \\
& Secondary analysis & $5(10.9)$ \\
& Mixed methods & $3(6.6)$ \\
& Qualitative research & $1(2.2)$ \\
\hline
\end{tabular}

*Multiple responses.

the articles published in CHNR have been survey-based. The proportion of literature reviews and secondary analysis studies, which comprised $13.2 \%$ of articles in 2014 [2] and $8.8 \%$ in 2016-2018 [1], increased to $23.9 \%$ in 2020 (literature reviews $13.0 \%$ and secondary analysis studies $10.9 \%$ ).

CHNR has increased the number of English publications each year, publishing one issue in English in 2018, two issues in 2019, and three issues in 2020. Furthermore, starting with the third issue in 2020, all articles are now published in English. The transition to an English-only publication will both make the journal more accessible for international readers and encourage submissions from international researchers. This change is also expected to promote the achievement of the journal's aim to contribute to the development of research, theory, and practice related to child health around the world, as well as in Korea, as an international journal. Furthermore, we have made incessant efforts to uphold the principles of transparency and best practice in scholarly publishing [3]. By complying with research ethics and by editing and publishing according to global standards through these efforts, we aim to be accepted for inclusion in PubMed Central, as well as maintaining the inclusion of CHNR in Scopus.

I am deeply grateful to both the reviewers who reviewed the manuscripts submitted to CHNR and the editors who edited papers even in the midst of the COVID-19 pandemic in 2020. It would not have been possible to publish the journal without the dedication and effort of our reviewers and editors. Thanks to the insightful perspectives and valuable comments of the reviewers and editors, we were able to publish highquality articles in CHNR. I express my sincere gratitude to all of you for your contributions to the publication and improvement of CHNR.

\section{Conflict of interest}

Hyun Young Koo has been editor-in-chief of Child Health Nursing Research since 2018. She was not involved in the review process of this editorial. No existing or potential conflict of interest relevant to this article was reported.

\section{Data availability}

Please contact the corresponding author for data availability.

\section{REFERENCES}

1. Im YJ. An analysis of research tendency in domestic and international academic journals of pediatric nursing. 2018 Winter academic conference of Korean Academy of Child Health Nursing; 2018 December 14; PJ Hotel. Seoul: Korean Academy of Child Health Nursing; 2018. p. 31-48.

2. Cho KC, Lee YE, Oh SE, Tak YR, Chae SM, Kim EJ, et al. Trend analysis of research articles published in Child Health Nursing Research 2014. Child Health Nursing Research. 2015;21(4):347-354. https://doi.org/10.4094/chnr.2015.21.4.347

3. Committee on Publication Ethics. Principles of transparency and best practice in scholarly publishing [Internet]. Eastleigh: Committee on Publication Ethics; 2018 [cited 2021 January 12]. Available from: https://doi.org/10.24318/cope.2019.1.12 